\title{
(6) OPEN ACCESS \\ Memantine for axial signs in Parkinson's disease: a randomised, double-blind, placebo-controlled pilot study
}

- Additional data are published online only. To view these files please visit the journal online (http://dx.doi. org/10.1136/jnnp-2012303182)

For numbered affiliations see end of article

\section{Correspondence to} Dr David Devos, Département de Pharmacologie Médicale, Université Lille Nord de France, CHRU de Lille, Lille F-59037, France;

david.devos@chru-lille.fr

Received 9 May 2012 Revised 28 August 2012 Accepted 3 September 2012 Published Online First 16 October 2012

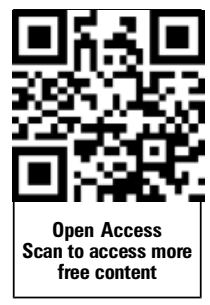

To cite: Moreau $C$, Delval A, Tiffreau $\mathrm{V}$, et al. J Neurol Neurosurg Psychiatry 2013:84: 552-555.

Caroline Moreau ${ }^{1,2}$ Arnaud Delval, ${ }^{1}$ Vincent Tiffreau, ${ }^{3}$ Luc Defebvre, ${ }^{1,2}$ Kathy Dujardin, ${ }^{1,2}$ Alain Duhamel, ${ }_{1}^{4}$ Gregory Petyt ${ }_{1}^{5}$ Claude Hossein-Foucher, ${ }^{5}$ David Blum, ${ }^{6}$ Bernard Sablonnière, ${ }^{6}$ Susanna Schraen, ${ }^{6}$ Delphine Allorge, ${ }^{7}$ Alain Destée, ${ }^{1,8}$ Régis Bordet, ${ }^{9,10}$ David Devos ${ }^{1,2,9}$

\begin{abstract}
Background Given that memantine is thought to decrease N-methyl-D-aspartic-acid-related (NMDA) glutamatergic hyperactivity and improve locomotion in rats, we sought to assess the drug's impact on axial symptoms in advanced Parkinson's disease (PD). Methods We performed a 90-day, randomised, double-blind, study with two parallel arms: $20 \mathrm{mg} /$ day memantine versus placebo (ClinicalTrials.gov: NCT01108029). The main inclusion criterion was the presence of a severe gait disorder and an abnormal, forward-leaning stance. The following parameters were analysed under standardised conditions before and after acute administration of L-dopa: gait (stride length as primary criterion), the United-Parkinson's-Disease-RatingScale (UPDRS) motor score and its axial subscore, the hypertonia and strength of the axial extensors and flexors (isokinetic dynamometer), the Dyskinesia Rating Scale score (DRS) and its axial subscore.

locomotion in a rat model of PD with bilateral PPN lesions. ${ }^{5}$ Memantine (1-amino-3,5-dimethyladamantane) is an uncompetitive, partial antagonist of the open NMDA receptor. ${ }^{6}$ By decreasing NMDA-dependant glutamatergic hyperactivity, memantine reduces akinesia and rigidity in the MPTP (1-methyl-4-phenyl-1,2,3,6-tetrahydropyridine) rat $\operatorname{model}^{7}$ and improves locomotion in rats treated with reserpine and $\alpha$-methyl-p-tyrosine. ${ }^{8}$

We hypothesised that decreasing excessive, NMDA-dependant, glutamatergic transmission might partly restore gait and posture control. In order to assess the feasibility of a multicentre clinical trial, we used sensitive measures and a standardised, acute L-dopa challenge to examine the impact of memantine on gait, axial motor symptoms and L-dopa-induced dyskinesia (LID) in advanced PD patients in a double-blind, placebocontrolled pilot study.
\end{abstract}

Results Twenty-five patients were included. The memantine and placebo group did not differ significantly in terms of stride length. However, in the memantine group, we observed significantly better results (vs placebo) for the overall UPDRS score $\left(\mathrm{F}_{(1,21)}=4.9\right.$; $p=0.039(-1))$ and its axial subscore $\left(F_{(1,21)}=7.2\right.$; $p=0.014(-1.1))$, axial hypertonia, the axial and overall DRS and axial strength.

Conclusions Memantine treatment was associated with lower axial motor symptom and dyskinesia scores but did not improve gait. These benefits must be confirmed in a broader population of patients.

\section{INTRODUCTION}

In late-stage Parkinson's disease (PD), axial signs (notably with gait disorders, such as hypokinesia), abnormal posture, falls and poor balance reduce personal independence and may prompt institutionalisation. These symptoms represent a public health issue for which a specific treatment is currently lacking. ${ }^{1}$ Dopaminergic depletion induces glutamatergic hyperactivity in the brain in general and in the subthalamic nucleus (STN) and its efferent pathways projecting to the pedunculopontine nucleus (PPN) in particular. ${ }^{2}{ }^{3}$ The PPN is particularly involved in posture and gait control. ${ }^{4}$

The N-methyl-D-aspartic-acid-related (NMDA) receptor antagonist MK-801 was found to facilitate

\section{PATIENTS AND METHODS}

The present randomised trial complied with the CONSORT 2010 guidelines and was registered at ClinicalTrials.gov (identifier: NCT01108029).

\section{Patients}

Consecutive patients (diagnosed according to Gibb's criteria ${ }^{9}$ and monitored at Lille University Hospital's PD clinic) were invited to participate in the study by $\mathrm{CM}, \mathrm{AD}, \mathrm{LD}$ and $\mathrm{DD}$ until the required number of participants was obtained. Patients were enrolled between May 2010 and November 2010, following their provision of written, informed consent to participation. The research protocol was approved by the local independent ethics committee (Protocol ID: 2008-008210-38). a severe gait disorder (defined as a score $\geq 2$ for UPDRS part III item 29) and an abnormal, forward-leaning stance (a score $\geq 2$ for item 28) despite optimal L-dopa treatment. The main exclusion criterion was the presence of axial disorders related to insufficient doses of L-dopa or offperiods of motor fluctuation or those induced by STN stimulation. Patients receiving STN stimulation had to have shown an improvement of at least $50 \%$ in motor symptoms during the first year of
The main inclusion criterion was the presence of 
this treatment (without a worsening in gait and posture). The appearance of axial signs immediately after the initiation of STN stimulation was also an exclusion criterion. Other notable exclusion criteria included (i) inability to walk unaided while on dopaminergic treatment, (ii) dementia (diagnosed according to the DSM-IV-R criteria and with a Mattis Dementia Rating Scale score $<130$ ) and (iii) the ongoing administration of an NMDA antagonist other than memantine.

\section{Experimental design}

We performed a 90-day, double-blind, placebo-controlled pilot study.

\section{Randomisation and masking}

Subjects were randomly assigned to memantine or placebo. The 1:1 assignment sequence (based on a computer randomnumber generator) was produced by our Department of Biostatistics. The randomisation list was sent to an independent contract research organisation (LC2, Lentilly, France) for preparation and distribution of identical capsules of memantine and placebo.

\section{Intervention}

Patients, study staff and investigators were blinded to the assignment. After a 30-day dose titration phase (with an increase of $5 \mathrm{mg}$ of memantine per week or a placebo), the patients received a daily dose (at 7:00) of $20 \mathrm{mg}$ of memantine (ie, the usual recommended dose) or placebo for a further 60 days. All patients undergoing STN stimulation were assessed under 'on-stim' conditions. Patients were not allowed to change their medication regimen or STN stimulation settings in the 3 months prior to the study or during the study itself.

\section{Efficacy criteria}

The primary efficacy criterion was the change in stride length (m) under 'on-L-dopa' conditions. Stride length was assessed in an optoelectronic analysis with a 6-camera VICON Video System from Oxford Metrics (Oxford, UK) (sampling rate: $50 \mathrm{~Hz})$. Secondary efficacy criteria included (i) gait velocity (m/ s) and cadence (steps/min); (ii) motor handicap, assessed as the overall UPDRS motor score and its axial subscore (the sum of items 18 (speech), 19 (facial expression), 22 (neck rigidity), 27 (arising from a chair), 28 (posture), 29 (gait) and 30 (postural stability)); (iii) LID, assessed as the overall Dyskinesia Rating Scale score and its axial subscore; (iv) hypertonia of axial flexors and extensors, assessed as the mean work (in joules) performed during 10 passive trunk movements at $30 \%$ on a CON-TREX isokinetic dynamometer (CMV AG, Dübendorf, Switzerland $)^{10}$; (v) trunk flexor and extensor strength, measured as the mean work (in joules) performed over three repetitions at $30 \%$ in active flexion and extension mode on the isokinetic dynamometer. ${ }^{10}$ All measurements were recorded in a doubleblind manner in our hospital's gait laboratory by CM, AD and VT.

\section{Standardised assessment}

Efficacy criteria were assessed at 8:30 in the morning under 'off-L-dopa' conditions, that is, at least $8 \mathrm{~h}$ after the withdrawal of dopaminergic medications. After acute administration of L-dopa at 9:00, the 'best on' condition was assessed at between 9:30 and 10:00 on the same morning. All assessments were performed once before the 90-day course of study medication and once afterwards. The L-dopa dose used in the assessment was $150 \%$ of the usual, first morning dose taken by patients to relieve their symptoms (table 1 ).

\section{Safety criteria}

Adverse events, arterial blood pressure values, an electrocardiogram and a standard blood biochemistry profile were recorded monthly. In view of a possible antagonistic effect of memantine on nicotinic acetylcholine receptors, ${ }^{5}$ drowsiness was assessed on the Epworth Sleepiness Scale. The study's Data and Safety Monitoring Board examined adverse event reports periodically but the blinding code was not broken. In order to estimate the final plasma concentration of memantine, a blood sample was taken from all patients (ie, both groups, to maintain blinding) before the morning administration of study medication at 7:00 on the last day of treatment.

\section{Sample size calculation}

In a previous study of 17 patients on methylphenidate, we had observed a stride length increase (relative to baseline) of $0.4 \mathrm{~m}$ (SD: 0.4 ) in the stand-walk-sit test after 3 months of treatment. ${ }^{11}$ Despite our use of a more sensitive optoelectronic analysis in the present study, we adopted the same anticipated stride length difference, that is, $0.4 \mathrm{~m}$ (SD: 0.4$)$. With a power of $80 \%$

Table 1 Characteristics of the study population

\begin{tabular}{llll}
\hline & Memantine & Placebo & Mann-Whitney test $p$ value \\
\hline Number of patients & 13 & 12 & $\mathrm{p}=0.68$ \\
Age at study entry (median [Q25-Q75]) (minimum-maximum) & $66[62-72](59-81)$ & $64[60-73](46-78)$ & $\mathrm{p}=0.27$ \\
Disease duration (years) (minimum-maximum) & $15[12-23](5-29)$ & $13.5[10-16.3](5-25)$ & $\mathrm{p}=0.83$ \\
Hoehn and Yahr score & $3[2.5-3]$ & $3[2.5-3]$ & $\mathrm{p}=0.89$ \\
L-dopa dose (mg) for 'on-L-dopa' assessments & $200[100-200]$ & $100[100-200]$ & $\mathrm{p}=0.93$ \\
L-dopa equivalent daily dose (mg) & $1000[700-1400]$ & $28[26-28.2]$ & $\mathrm{p}=0.79$ \\
Mini mental scale examination score & $28[26-28.2]$ & $136[132-139]$ & $\mathrm{p}=0.85$ \\
Mattis dementia rating scale score & $137[130-139]$ & $9[5-10]$ & $\mathrm{p}=0.53$ \\
Drowsiness on the Epworth sleepiness scale & $8[4-9]$ & $8.5[3-14]$ & $\mathrm{p}=0.69$ \\
Montgomery-Asberg depression rating scale & $8[6-16]$ & $\mathrm{n}=8$ of 12 & $7.5[5.5-8.25]$ \\
Patients with subthalamic nucleus stimulation & $\mathrm{n}=8$ of 13 & $95[74-126]$ & $\mathrm{p}=0.44$ \\
Stimulation duration (years) & $8[7.25-9]$ & $85[70-131]$ & $\mathrm{p}=0.41$
\end{tabular}

The parameters are expressed as the median value [1st quartile-3rd quartile]. The groups were similar at baseline. The total electrical energy delivered (TEED) was calculated as $\left(V^{2 *}\right.$ frequency* pulse width $) / 2$ 
and a type I error of $5 \%$, the total sample size was found to be 17 patients per group. Next, on the basis of (i) a coefficient of 0.4 for the correlation between the baseline measurement and the end-of-study measurement in a covariance analysis, (ii) a very low dropout rate and (iii) the replacement of dropouts, we calculated the required sample size to be 14 patients per group.

\section{Statistical analysis}

Descriptive analyses and the Shapiro-Wilk test were used to check whether data were normally distributed. Non-normally distributed data were log-transformed. The primary criterion was tested in a covariance analysis (with adjustment for baseline) for all patients with data recorded in the final assessment. The effect size was also computed. Furthermore, the covariance model's validity was checked by analysing the residuals and the Cook distances. Numerical safety data for all randomised patients were assessed in an analysis of variance. The threshold for statistical significance was set to $p=0.05$ in all cases. All statistical tests were two-tailed and performed with SAS software (V.9.2, SAS Institute Inc., Cary, North Carolina, USA).

\section{RESULTS}

We prospectively enrolled 25 patients with a severe gait disorder and an abnormal, forward-leaning stance. Three patients dropped out due to lack of efficacy (placebo: $n=2$; memantine: $n=1$ ) but two of the latter (one in each group) were included in the final efficacy analysis because they had dropped out shortly before the end of the study (see the see online supplementary figure (flowchart) and supplementary data on the criteria for ending recruitment). On the basis of interviews with the patients and caregivers and a monthly pill count, the treatment compliance was above $90 \%$ for all patients. The median plasma concentration of memantine was $83 \mathrm{ng} / \mathrm{ml}$ (76.5-98.3).

\section{Efficacy criteria}

The 'off-L-dopa' condition could not be studied, since 18 patients with very advanced PD were unable to walk unaided when L-dopa had been withdrawn. Memantine's effects in the 'on-L-dopa' condition are presented in the table 2.

\section{Safety criteria}

There were no significant safety differences between the memantine and placebo groups. Other than a slight worsening of pre-existing alopecia in one female patient, no adverse events were reported. There was no significant change in drowsiness over the course of the memantine treatment.

\section{DISCUSSION}

Our randomised, double-blind, placebo-controlled pilot study showed for the first time that a combination of memantine and L-dopa was associated with a slight, beneficial effect on axial motor handicap and LID in advanced PD patients with severe axial symptoms. Memantine's good safety profile and its observed association with a lower motor symptom score (vs placebo) confirmed the findings of two open-label studies ${ }^{12} 13$ and two double-blind, placebo-controlled studies. One of the double-blind studies used non-validated scales ${ }^{14}$ and the other adopted a crossover paradigm with a very small number $(n=12)$ of PD patients. ${ }^{15}$

This pilot study had both strengths and limitations. The main limitation was the small sample size, which encompassed patients with and without STN stimulation. However, the lack of significant differences between these subgroups suggests that memantine may have clinical benefit in both stimulated and non-stimulated patients. Furthermore, the lack of 'off-L-dopa' data (due to handicap in patients with very advanced disease) prevented us from interpreting the effect of memantine in the absence of L-dopa. In contrast, the study had a number of key strengths: it featured a double-blind, placebo-controlled design

Table 2 Efficacy criteria during standardised 'on-L-dopa' assessment

\begin{tabular}{|c|c|c|c|c|c|}
\hline & \multicolumn{2}{|c|}{ Memantine ( $n=13)$} & \multicolumn{2}{|l|}{ Placebo $(n=11)$} & \multirow{2}{*}{$\begin{array}{l}\text { p-value (adjusted effect size) } \\
\text { Covariance analysis }\end{array}$} \\
\hline & Study entry & Study end & Study entry & Study end & \\
\hline \multicolumn{6}{|l|}{ Gait (optoelectronic analysis) } \\
\hline Stride length $(\mathrm{m})$ (increased=better) & $1.09[1.02-1.2]$ & $1.05[0.93-1.2]$ & $0.84[0.77-1.18]$ & $1.12[0.75-1.24]$ & $F_{(1,21)}=0.27 ; p=0.61(-0.2)$ \\
\hline Velocity (m/s) (increased=better) & $0.99[0.95-1.1]$ & $1.01[0.76-1.07]$ & $0.81[0.69-1.06]$ & $1.05[0.75-1.12]$ & $F_{(1,21)}=0.54 ; p=0.47(-0.3)$ \\
\hline Cadence (steps $/ \mathrm{min}$ ) (decreased=better) & 114 [108-127] & 112 [99-124] & 112 [106-116] & 114 [109-114] & $F_{(1,21)}=0.72 ; p=0.41(-0.4)$ \\
\hline \multicolumn{6}{|l|}{ Motor handicap (motor UPDRS score) } \\
\hline Axial subscore & $10[8-12]$ & $9[6-10]$ & $10[8-13]$ & $10[8-13]$ & $F_{(1,21)}=7.2 ; p=0.014(-1.1)$ \\
\hline Overall score & 26 [19-37] & $25[15-34]$ & $26[22-35]$ & 27 [23-35] & $F_{(1,21)}=4.9 ; p=0.039(-1)$ \\
\hline \multicolumn{6}{|l|}{ Dyskinesia (Dyskinesia Rating Scale) } \\
\hline Axial subscore & $3[2-3]$ & $1[0-2]$ & $3[1-4]$ & $3[1-3.5]$ & $F_{(1,21)}=11 ; p=0.003(-1.4)$ \\
\hline Overall score & $5[3-7]$ & $3[1-5]$ & $6[1-9]$ & $6[1-10]$ & $F_{(1,21)}=19 ; p=0.0003(-1.9)$ \\
\hline \multicolumn{6}{|c|}{ Trunk hypertonia (isokinetic dynamometer) (decrease=better) } \\
\hline Flexor $(J)$ & $4.3[3.5-6.6]$ & $3.1[2.3-4.7]$ & $4.4[3.2-7.2]$ & $4.9[4-7]$ & $F_{(1,18)}=21.3 ; p=0.0001(-2)$ \\
\hline Extensor $(\mathrm{J})$ & $3.9[2-8.5]$ & $2.6[1.9-6.5]$ & $4.6[2.7-5.1]$ & $5[4.1-5.4]$ & $F_{(1,18)}=12.5 ; p=0.002(-1.5)$ \\
\hline \multicolumn{6}{|c|}{ Trunk flexor and extensor strength (isokinetic dynamometer) (increase=better) } \\
\hline Flexor $(J)$ & $25[18-26]$ & $31[25-37]$ & 27 [15-32] & $26[17-31]$ & $F_{(1,18)}=13.5 ; p=0.002(1.8)$ \\
\hline Extensor $(\mathrm{J})$ & 25 15-31] & $33[25-39]$ & $21[15-36]$ & $21[17-33]$ & $F_{(1,18)}=12.8 ; p=0.02(1.7)$ \\
\hline
\end{tabular}


and (in order to compensate for the small sample size) highly sensitive measurement techniques under standardised assessment conditions.

The memantine and placebo group did not differ significantly in terms of stride length (the study's primary efficacy criterion) and other gait parameters assessed with a sensitive optoelectronic system. The small observed effect size suggests that gait might not even be improved in future studies with a larger sample size population. We also failed to detect any significant differences (vs placebo) in attention (as assessed by measuring reaction times: data not shown) or sleepiness in patients taking their usual dopaminergic medication.

Axial motor signs (as judged by the UPDRS axial subscore) were significantly lower in the memantine group than in the placebo group. This clinical benefit was associated with an improvement in axial rigidity and strength, as measured with an isokinetic dynamometer. Relative to placebo, both LID of the limbs and axial LID were less intense in the memantine group. Little is known about memantine's effect on LID-in contrast to amantadine, another NMDA receptor antagonist-because placebo-controlled studies on this topic are lacking.

The beneficial effect of memantine may be due to a decrease in the excessive synaptic noise caused by overactivation of NMDA receptors-notably those in the descending subthalamo-entopeduncular pathway. ${ }^{2-4}$ This short-term effect might have a favourable longer-term impact on posture. Memantine might decrease trunk flexor rigidity (limiting the abnormal, forward-leaning stance) and increase axial extensor strength (limiting extensor under-use and thus slowing the development of the progressive amyotrophy that notably occurs in parkinsonian camptocormia).

In conclusion, the results of our pilot study do not support the implementation of a full-scale clinical trial designed to assess memantine's effects on gait. However, memantine's potential benefit on other axial motor symptoms and dyskinesia should be confirmed in a larger patient population.

\footnotetext{
Author affiliations

${ }^{1}$ Department of Neurology, University of Lille Nord de France and Lille University Hospital, Lille, France

${ }^{2}$ EA4559, University of Lille Nord de France and Lille University Hospital, Lille, France

${ }^{3}$ EA 4488, Department of Rehabilitation Medicine, University of Lille Nord de France and Lille University Hospital, Lille, France

${ }^{4}$ Department of Biostatistics, University of Lille Nord de France and Lille University Hospital, Lille, France

${ }^{5}$ Department of Nuclear Medicine, University of Lille Nord de France and Lille University Hospital, Lille, France

${ }^{6}$ Department of Molecular Biology, INSERM U837/1 JPARC, University of Lille Nord de France and Lille University Hospital, Lille, France

${ }^{7}$ Department of Biology and Toxicology, University of Lille Nord de France and Lille University Hospital, Lille, France

${ }^{8}$ INSERM U837/6 JPARC, University of Lille Nord de France and Lille University Hospital, Lille, France

${ }^{9}$ Department of Medical Pharmacology, University of Lille Nord de France and Lille University Hospital, Lille, France

${ }^{10}$ Department of Pharmacology, EA 1046, University of Lille Nord de France and Lille University Hospital, Lille, France
}

Acknowledgements The authors wish to thank Lundbeck for kindly providing the memantine and the placebo, the Fédération de la Recherche Clinique du CHU de Lille (Lille University Hospital) for promoting the study, Dr David Fraser (Biotech Communication, Damery, France) for improving the manuscript's English and Marie Delliaux and Florence Beaucamp for data acquisition. The authors also wish to thank the study assistants Valerie Vasseur, Carine Piatek and Francine Niset.
Funding This academic study was funded by a PHRC grant from the French Ministry of Health. This work was supported by Projet Hospitalier National de Recherche Clinique in 2008 with grant number 2008-008210-38

Contributors CM and AD: research project: conception, organisation, execution; and manuscript: writing of the first draft. LD and KD: research project: conception, organisation, execution; and manuscript: review and critique. VT, CH-F, GP, DB, SS and DA: research project: execution; and manuscript: review and critique. AD carried out the biostatistical analysis. AD: manuscript: review and critique. RB: research project: conception, organisation; and manuscript: review and critique. DD: research project: conception, organisation, execution; and manuscript: writing of the first draft, review and critique and carried out the biostatistical analysis. The principal investigator (DD) had full access to all the study data and had final responsibility for submitting the study report for publication.

\section{Competing interests None.}

Patient consent Obtained.

Ethics approval Local independent ethics committee (Protocol ID: 2008-008210-38).

Provenance and peer review Not commissioned; externally peer reviewed.

Open Access This is an Open Access article distributed in accordance with the Creative Commons Attribution Non Commercial (CC BY-NC 3.0) license, which permits others to distribute, remix, adapt, build upon this work non-commercially, and license their derivative works on different terms, provided the original work is properly cited and the use is non-commercial. See: http://creativecommons.org/ licenses/by-nc/3.0/

\section{REFERENCES}

1 Bloem BR, Grimbergen YA, Cramer M, et al. Prospective assessment of falls in Parkinson's disease. J Neurol 2001;248:950-8.

2 Rodriguez MC, Obeso JA, Olanow CW. Subthalamic nucleus-mediated excitotoxicity in Parkinson's disease: a target for neuroprotection. Ann Neurol 1998:44(3 Suppl 1):S175-88.

3 Carroll CB, Holloway V, Brotchie JM, et al. Neurochemical and behavioural investigations of the NMDA receptor-associated glycine site in the rat striatum: functional implications for treatment of parkinsonian symptoms. Psychopharmacology (Berl) 1995;119:55-65.

4 Pahapill PA, Lozano AM. The pedunculopontine nucleus and Parkinson's disease. Brain 2000;123:1767-83.

5 Steiniger B, Kretschmer BD. Effects of ibotenate pedunculopontine tegmental nucleus lesions on exploratory behaviour in the open field. Behav Brain Res 2004:151:17-23.

6 Johnson JW, Kotermanski SE. Mechanism of action of memantine. Curr Opin Pharmacol 2006:6:61-7.

7 Kucheryanu VG, Kryzhanovskii GN. Effect of glutamate and antagonists of $\mathrm{N}$-methyl-D-aspartate receptors on experimental parkinsonian syndrome in rats. Bull Exp Biol Med 2000;130:629-32.

8 Skuza G, Rogoz Z, Quack G, et al. Memantine, amantadine, and L-deprenyl potentiate the action of L-dopa in monoamine-depleted rats. J Neural Transm Gen Sect 1994:98:57-67.

9 Gibb WR, Lees AJ. The relevance of the Lewy body to the pathogenesis of idiopathic Parkinson's disease. J Neurol Neurosurg Psychiatry 1988:51:745-52.

10 Pang MY, Mak MK. Trunk muscle strength, but not trunk rigidity, is independently associated with bone mineral density of the lumbar spine in patients with Parkinson's disease. Mov Disord 2009:24:1176-82.

11 Devos D, Krystkowiak P, Clement F, et al. Improvement of gait by chronic, high doses of methylphenidate in patients with advanced Parkinson's disease. J Neurol Neurosurg Psychiatry 2007;78:470-5.

12 Fischer PA, Jacobi $\mathrm{P}$, Schneider $\mathrm{E}$, et al. Effects of intravenous administration of memantine in parkinsonian patients (author's transl). Arzneimittelforschung 1977:27:1487-9

13 Schneider E, Fischer PA, Clemens R, et al. Effects of oral memantine administration on Parkinson symptoms. Results of a placebo-controlled multicenter study. Dtsch Med Wochenschr 1984:109:987-90.

14 Rabey JM, Nissipeanu P, Korczyn AD. Efficacy of memantine, an NMDA receptor antagonist, in the treatment of Parkinson's disease. J Neural Transm Park Dis Dement Sect 1992;4:277-82

15 Merello M, Nouzeilles MI, Cammarota A, et al. Effect of memantine (NMDA antagonist) on Parkinson's disease: a double-blind crossover randomized study. Clin Neuropharmacol 1999:22:273-6. 